\title{
Arbor
}

\section{Atención y relato}

\author{
Montserrat González
}

Arbor CLXVII, 697 (Enero 2004), 81-104 pp.

El relato como modelo de construcción cognitiva que se desarrolla a lo largo de un eje temporal ha sido utilizado ampliamente por lingüistas funcionales y cognitivistas, narratólogos y estudiosos del procesamiento del discurso, en general. Los hechos conforman el esqueleto de una narración, pero la forma en que éstos se organizan, combinados con la orientación espaciotemporal, su descripción y su evaluación resulta en una percepción e interpretación determinada de los mismos. En el proceso cognitivo de comprensión del relato, la tradición jerárquica trata de dar cuenta de los esquemas y modelos o estructuras mentales que se construyen. En esta tradición, el hecho de que un hablante escoja una determinada forma lingüistica para contar una historia tiene un efecto directo en la forma en que el oyente entiende, o procesa, los acontecimientos. El objetivo de este articulo es presentar la utilización, por parte del hablante, de ciertas marcas lingüisticas que hacen esto último posible. Dentro de los mecanismos lingüísticos que nos facilitan el procesamiento de la información que recibimos, encontramos los deicticos (pronombres, determinadas formas verbales de tiempo, adjetivos y adverbios), las cláusulas (principales y subordinadas), los conectores argumentativos y los marcadores pragmáticos. Su función es facilitar el procesamiento de la información y ayudar al oyente a centrar su atención en determinadas partes del discurso, funcionando como ejes focalizadores. En general, son señales que anuncian un cambio de foco inminente, un movimiento que el hablante dificilmente puede anticipar y predecir. Todo ello se verá ejemplificado en dos tipos de relato oral: la historia de la rana Frog, where are you? (Mayer 1969) y el relato de experiencia personal (Labov y Waletzky 1967, Labov 1972). 
Gran parte de la bibliografía que trata de cómo se procesa el discurso por parte del oyente o del lector utiliza el relato como modelo de construcción cognitiva que se desarrolla a lo largo de un eje temporal (Schegloff 1982, Bamberg y Marchman 1991, Segal et al. 1991). En este sentido, la construcción de un relato se convierte en un objeto interactivo dinámico que, poco a poco, va tomando forma, hasta llegar al texto final. Su elaboración implica la integración de dos procesos cognitivos: uno llamado referencial, que consiste en la secuenciación de los acontecimientos siguiendo un patrón conceptual, lingüísticamente codificado en frases y oraciones, y otro llamado jerárquico, que ordena dichas unidades lingüísticas siguiendo una pauta temática, con una visión más globalizadora que la que implica el anterior. La tradición referencial del relato se centra en los datos de producción e investiga la forma en que los estados y los acontecimientos se actualizan en el texto oral, concentrándose en las formas lingüísticas y en los enlaces que hacen que el discurso sea cohesivo. Por el contrario, la tradición jerárquica trata de dar cuenta de los esquemas y modelos o estructuras mentales que se construyen en el proceso cognitivo de comprensión del relato. En esta última tradición, el hecho de que un hablante escoja una determinada forma lingüística para contar una historia tiene un efecto directo en la forma en que el oyente entiende, o procesa, los acontecimientos. El objetivo de este artículo es presentar la utilización, por parte del hablante, de ciertas marcas lingüísticas que hacen esto último posible. En el primer apartado me ocuparé de esta cuestión. La segunda parte estará dedicada a las marcas lingüísticas de 'focalización' y/o de 'atención' en el relato, marcas que ayudan al hablante a organizar la información y dan pautas al oyente para que procese aquello que considera más relevante, esto es, aquella parte del relato -palabra, oración o segmentoque merece una atención especial. En esta parte se tratará el tema de la focalización (focus o ground) a través de los deícticos, la referencia anafórica, las cláusulas (subordinadas y principales) y los marcadores del discurso. Todo ello se hará ejemplificado en dos modelos de relato oral: la historia visual de Frog, where are you? (Mayer 1969) y el modelo de relato oral laboviano de experiencias personales (Labov y Waleztky 1967, Labov 1972). En la tercera parte profundizaré en ciertas marcas lingüísticas de procesamiento del discurso, los marcadores pragmáticos, haciendo una mención especial a aquéllos que guían al oyente a través de los procesos inferenciales. Finalmente, mencionaré las diferencias retóricas del relato en español y en inglés, en relación al tema que nos ocupa. 


\section{El relato oral. Aproximación discursiva y cognitiva}

Cuando construimos un relato oral, lo hacemos teniendo en cuenta unos esquemas formales, de contenido lingüístico, y unos esquemas funcionales, donde la estructuración e interpretación de lo que contamos adquiere un papel fundamental. Así, utilizamos el código lingüístico para contar una historia, pero es muy importante cómo la organizamos y transmitimos a nuestro interlocutor. Bamberg y Marchman (1991) lo explican de la siguiente forma:

"The choice of lexical or grammatical forms not only represents the speaker-event (referential) relationship, but at the same time the speaker-hearer (pragmatic) relationship, that is, it signals not only what we talk about, but at the same time how we talk about it, that is, how we want (what we talk about) to be understood." (1991:279)

La apreciación de Bamberg y Marchman es altamente pertinente para el estudio del relato oral ya que implica que siempre que un hablante escoge una determinada forma lingüística para contar una historia, ello tiene un efecto directo en cómo el oyente la interpreta. Sin embargo, no existe una forma preconcebida de contar un relato, donde los acontecimientos, los participantes y los procesos cognitivos estén preestablecidos, como entidades independientes de las cuales las historias, los argumentos y las emociones surgen. No existe, pues, un producto a priori; existe un proceso dinámico de creación que resulta en un producto, en este caso en particular, en un relato. Desde un punto de vista cognitivo, existe así una simultaneidad de forma y función, esto es, una simultaneidad entre la utilización de formas que, conceptualmente, hacen posible el hilo argumentativo de la historia y la organización global del relato, donde las emociones, la expresividad y la intencionalidad del narrador se plasma y hace posible que dos relatos orales, sobre un mismo tema o argumento, no sean nunca iguales ${ }^{1}$. Este punto de vista, que sigue una tradición de estructura jerárquica del relato, en un marco claramente cognitivo, es ampliamente aceptado por numerosos estudiosos del género, desde los que estudian la adquisición del relato desde la infancia (Bamberg y Reilly 1996, por ejemplo), hasta los que lo hacen desde la lingüística computacional o la inteligencia artificial (Grosz y Sidner 1986, Sanders 1997, entre otros). De acuerdo con Aksu-Koç (1996), los individuos interpretamos y experimentamos el mundo que nos rodea de forma colectiva, dentro de ciertos marcos o esquemas mentales determinados por el individuo y por variables socioculturales. Según este autor, existe, sin embargo, un denominador común en la forma de contar una historia, un esquema universal-que 
incluye un principio, un conflicto o desarrollo de la acción y un resultado- y una organización secuencial de los acontecimientos que hace que éstos sean adecuadamente interpretados (Aksu-Koç 1996: 310). Siguiendo la misma pauta que Bamberg y Marchman, Aksu-Koç nos remite, también, a dos niveles de estructuración, uno lineal, de ordenación temporal de los acontecimientos, y otro temático, donde los episodios se organizan de forma jerárquica de acuerdo con la macroestructura temática de la historia. El narrador puede presentar los hechos bajo distintos puntos de vista y voces, ofreciendo una estructura que contiene diferentes niveles de lectura: la del narrador, en primera persona, avanzando en el relato al mismo tiempo que avanzan los hechos o la acción, o como un personaje más de la historia que experimenta los acontecimientos desde fuera. El narrador tiene, así, la posibilidad de tener más de una voz en el relato:

«The intersection of different voices and shifts between them allows the teller to recount an event from within the narrative line and to comment on it from without at the same time, thus contributing to the weaving of a rich and colourful story.» (AksuKoç 1996:310)

$\mathrm{Si}$ a la propiedad polifónica que presenta generalmente el relato se le añade las propiedades de un modelo mental, nos encontramos con una aproximación claramente cognitiva. Segal et al. (1991) han denominado 'mental model-deictic shift view' a un modelo mental que combina propiedades de las dos tradiciones anteriormente mencionadas, la local -con secuenciación lineal de los acontecimientos y conceptualización de los hechos- y la global, donde entran en juego la organización, punto de vista e intencionalidad del hablante, y organización temática según determinados esquemas mentales ${ }^{2}$. En su propuesta de modelo, una vez dentro del mundo del relato, el oyente o lector puede cambiar de un determinado centro referencial a otro a través de los personajes, el tiempo y el lugar, creando puentes entre el creador del relato, esto es, entre el narrador, y el lector u oyente de éste (p. 31). Los autores lo ejemplifican a través del uso de conectores interclausales que ayudan a construir un determinado modelo mental.

Los modelos cognitivos y referenciales de interpretación y comprensión del lenguaje, y en el caso que nos ocupa del relato, intentan dar cuenta de la presencia de ciertas unidades lingüísticas que facilitan la integración de gran cantidad de información y que lo hacen estructurándola según su grado de relevancia para el oyente o el lector. En el siguiente apartado veremos los mecanismos lingüísticos más destacados que utiliza, para tal fin, el narrador. 


\section{Mecanismos lingüísticos de «focalización» y/o de «atención» en el relato}

Según Langacker (1991), el conocimiento lingüístico del hablante más que de carácter declarativo, es de tipo procedimental. Utilizamos el código lingüístico para transmitir información pero vamos más allá de las condiciones de verdad-no verdad de aquello que transmitimos. El hablante utiliza formas léxicas que facilitan y/o restringen la realización de inferencias por parte del oyente; éste, a su vez, crea una relación entre aquello que se le comunica y el contexto (situacional pero, sobre todo, cognitivo, de conocimiento compartido y de conocimiento del mundo en general). La gramática cognitiva postula que el estudio del lenguaje no puede hacerse independientemente de su función cognitiva y comunicativa, y que el lenguaje tiene un carácter simbólico cuya función primordial es la de significar. La función de la gramática es, pues, estructurar y simbolizar el contenido semántico (Cuenca y Hilferty 1999:19). Si trasladamos este marco conceptual al tema que nos ocupa, observamos que, como usuarios de un código lingüístico, recurrimos constantemente a una serie de unidades léxicas que nos ayudan a procesar el discurso. Estas unidades pertenecen a categorías gramaticales diversas, que se agrupan, a su vez, en grandes categorías funcionales: pronombres y adverbios que funcionan como deícticos referenciales o temporales, por ejemplo, o sintagmas preposicionales o adverbiales que, desde una perspectiva funcional, son conectores interclausales. Veámoslo ejemplificado en (1). Portolés (1998) nos muestra la función que desempeñan por tanto y sin embargo, ambos conectores interclausales. Como acertadamente señala Portolés, ambos conectores no aportan ningún valor de verdad al significado proposicional de la oración; su función se ciñe a restringir y acotar una serie de inferencias (p. 22).

(1)

a) Ana es de Teruel y, por tanto, es habladora.

b) Ana es de Teruel y, sin embargo, es habladora

Tanto en a) como en b), el contenido proposicional es el mismo: Ana es de Teruel y es habladora. Sin embargo, a) y b) no significan lo mismo ya que las inferencias que se derivan al utilizar por tanto y sin embargo son distintas: en el primer caso establecemos un silogismo; si los habitantes de Teruel son habladores y Ana es de esta ciudad, Ana es habladora. Por el contrario, lo que inferimos en el segundo caso es que Ana es habladora, aunque los turolenses no lo sean (Portolés 1998:22).

$\mathrm{Al}$ tratar mecanismos lingüísticos que nos facilitan el procesamiento de la información que recibimos, cabe tener en cuenta una serie de con- 
ceptos que nos ayudan a enmarcar y entender su operatividad. Así, dentro del ámbito cognitivo, hablamos de los deícticos como expresiones que necesariamente invocan o se refieren al acontecimiento de habla (the speech event), a sus participantes y a sus circunstancias inmediatas, tales como el tiempo y el lugar del suceso. La lingüística cognitiva se refiere a estos elementos como elementos de anclaje (grounding elements o ground $^{3}$ ) . Veamos cómo Langacker (1991) nos explica cómo todos ellos están presentes en la expresión lingüística:

"The very fact of being a linguistic expression implies a potential user, and in any actual use the speaker and hearer are likely to be at least dimly aware of their role in entertaining and construing the conception evoked.» (1991: 318)

Según Langacker, cuando nos referimos a dichos elementos con un alto grado de subjetividad (por ejemplo, al utilizar pronombres personales en primera persona), lo que hacemos es incluirlos dentro de la 'perspectiva' o 'punto de vista' del hablante (1991: 322). Sin embargo, estos conceptos requieren cierta elaboración ya que lo que significan para algunos autores recibe un término distinto para otros. Así, Berman y Slobin (1994) hablan de 'perspectiva' como una categoría funcional que recurre a formas de expresión lingüística relacionadas con el tema y la información más o menos relevante. En inglés, dichas expresiones incluyen verbos en voz activa o pasiva, variación del orden de las palabras en la oración, referencia pronominal y nominal, conectores y marcadores del discurso. En lugar de utilizar el término 'perspectiva', Toolan (1988) se refiere a 'focalización' como un concepto que implica la adopción de una perspectiva (lo sitúa en el relato, en particular), «a viewpoint from which things are seen, felt, understood, assessed.» (p. 68). En su caso, los deícticos (que abarcan pronombres personales, distintas formas verbales de tiempo, adjetivos y adverbios) funcionan como signos que indican al oyente que el discurso está 'anclado', asentado, con referencias claras en cuanto a la situación temporal y espacial de los participantes. Los términos 'focalización' y 'punto de vista', señala Toolan, son ampliamente utilizados entre los autores angloamericanos; él prefiere utilizar el término 'orientación', ya que, en su opinión, éste es menos visual y más adecuado para una perspectiva cognitiva del lenguage, la cual incluye, señala el autor, el ámbito emotivo e ideológico (1988: 68). En relación al relato, Toolan lo ejemplifica con el hecho de que la voz del que habla, piensa y describe puede o no coincidir con un único personaje. Asimismo, éste puede situarse dentro y fuera de la historia. Si lo hace desde el exterior, no lo hará ligado a ningún personaje en particular; si lo hace desde el interior, implicado en los acontecimientos, tomará una postura determinada, posiblemente. 
El 'punto de vista', la 'perspectiva', la 'focalización' o la 'orientación' -el uso de los términos no es realmente lo más importante; sí lo es su conceptualización- de lo que en lingüística cognitiva se denomina ground nos lleva, en el terreno lingüístico, a ser más precisos en lo que se refiere a la utilización de fórmulas que nos acerquen o nos alejen de la situación, los hechos y los participantes. Los hablantes utilizamos distintos mecanismos lingüísticos que facilitan o no la inclusión del sujeto dentro del enunciado, de la situación y de los hechos que se están narrando, en el caso del relato. Podemos distanciarnos o podemos involucrarnos totalmente en aquello que estamos contando o describiendo. En términos cognitivistas, se utiliza los términos foreground y background para referirse a aquella información que situamos en un 'primer plano' (traducción literal del término, aunque suficientemente esclarecedora) y aquélla que nos sirve como escenario de fondo, donde los hechos y los participantes transcurren. Según Berman y Slobin (1994) las marcas lingüísticas que utilizamos los hablantes para anclar al oyente (como ground), son marcas que guían su atención. Cada lengua utiliza fórmulas distintas. El grado más o menos alto de atención requerido lo controlará el narrador, sostienen Berman y Slobin (1994: 7). En algunas lenguas, como el ruso, los narradores utilizan el aspecto verbal y las cláusulas subordinadas para conseguir efectos dramáticos ${ }^{4}$. Berman y Slobin hacen referencia a cómo Labov (1972) y Hopper (1979) hacen uso de distintas formas lingüísticas con una función muy determinada (p. 7). Según Labov, las cláusulas subordinadas no ayudan a avanzar en el relato, mientras que las principales sí lo hacen. Estas incluyen verbos en forma activa, en tiempo perfectivo, sujetos animados, orden canónico de las partes de la oración y marcas temáticas. Todo ello lo encontraríamos en la información más destacada, en el foreground. Por el contrario, los verbos en imperfecto y las cláusulas no canónicas (pasivas, subordinadas, etc.), las encontraríamos en el background, en el trasfondo de la acción. Hopper incluye las marcas de tiempo verbal en aquella parte del relato más dinámica, concisa, donde ocurren los hechos más significativos. En un segundo plano, encontraríamos las marcas de aspecto verbal, transmitiendo los comentarios, la descripción de los estados físicos o anímicos. Finalmente, Berman y Slobin señalan que es el narrador el que escogerá qué hechos o elementos destacar en el relato y, por tanto, será él quien decidirá qué formas lingüísticas utilizar:

"... foreground and background are only partially determined by an objective logic of
events. They are also the product of creative acts of perspective-taking, by which the
narrator guides the listener in a subjective interpretation of the causal/motivational
network of events, assigning salience to particular points.» (Berman y Slobin 1994: 9) 
El relato oral requiere un alto grado de atención por parte del oyente. Éste, no sólo quiere saber qué pasó, cuáles fueron los hechos, cómo acabó la historia y qué grado de implicación tiene o siente que tiene el narrador, sino que, inconscientemente, hace todo lo posible por mostrarse atento y formar, él mismo, parte de la historia. El oyente siente que ha de formar parte del mundo del relato y más si se trata de un relato de experiencia personal, como es el caso de los que nos ofrecen Labov y Waletzky (1967), con una clara y bien definida estructuración de la situación, geográfica y espaciotemporal, de los hechos, de los participantes, de lo que ocurrió y del desenlace, incluyendo una evaluación más o menos subjetiva del narrador, implicado al cien por cien en contar su experiencia personal a un interlocutor. El narrador es consciente del alto grado de atención del oyente y le facilita el camino, o el proceso cognitivo, a través del cual llegará a una interpretación de los hechos. Las formas lingüísticas que utiliza para tal fin son, por tanto, altamente significativas. Los deícticos funcionan como elementos que el hablante utiliza para guiar, captar o centrar la atención del oyente en aquella parte del relato que tiene que ver con la situación, los hechos y los participantes (ground). Las formas verbales que indican temporalidad, las cláusulas -principales o subordinadas- y los conectores y marcadores del discurso tienen también una función significativa. En el siguiente apartado veremos la utilización de algunas de estas formas lingüísticas ejemplificadas en dos tipos de relato oral: la entrevista sociolingüística de experiencia personal (Labov y Walezky 1967, Labov 1972), y la conocida historia de la rana -Frog, where are you? (Mayer 1969)- ampliamente utilizada en el ámbito psicolingüístico para el estudio de adquisición de primeras lenguas, principalmente.

\section{Marcas de atención en dos casos de relato oral: relato laboviano y Frog, where are you?}

La organización de los hechos, de lo que acontece en un relato oral, ha sido ampliamente estudiado y analizado por lingüistas funcionales y cognitivistas, narratólogos y estudiosos del procesamiento del discurso, en general. Los hechos conforman el esqueleto de una narración. La forma en que se organizan, combinados con la orientación espaciotemporal, su descripción y su evaluación -subjetiva y objetiva- resulta en una percepción e interpretación determinada de los mismos. El narrador puede contarlos en tiempo pasado, si se trata de una experiencia personal, o en tiempo presente si está describiendo lo que ocurre en una secuencia de viñetas sin texto, por ejemplo. 
La entrevista sociolingüística laboviana de experiencia personal (Labov y Waletzky 1967, Labov 1972) elicita una narración a través de preguntas que, según Labov, resultan altamente productivas. Dos de las más utilizadas son: «Alguna vez has sentido que estabas en peligro de muerte?» y "Alguna vez has tenido una premonición, has tenido la sensación de que algo iba a ocurrir?». Labov sostiene que todos los seres humanos hemos tenido, alguna vez, este tipo de sensaciones y que, por tanto, todos tenemos algo que contar. $\mathrm{Al}$ responder a una pregunta de este tipo, Labov observó que los entrevistados mantenían un patrón regular de estructuración del relato: empezaban con un breve resúmen de la historia (abstract), orientaban al interlocutor en cuanto a personajes involucrados, tiempo en el que ocurrieron los hechos, y situación geográfica de los mismos (orientation), narraban los hechos (developing of action) y finalizaban con unas frases que explicaban el resultado de la historia, cómo acabó todo (result). Entre medio, evaluaban lo ocurrido, su estado de ánimo (internal evaluation), e introducían información que consideraban relevante para el interlocutor (aclaraciones, por ejemplo, o información adicional: external evaluation). En algunos casos, la historia se cerraba con 'y esto es lo que ocurrió' (coda). Veamos en (2) un ejemplo de relato de experiencia personal en inglés. Responde a la primera pregunta, de situación de peligro ${ }^{5}$. Está segmentado siguiendo el modelo laboviano $^{6}$.

(2)

\begin{tabular}{|c|c|c|}
\hline \multicolumn{3}{|c|}{$@$ Begin } \\
\hline \multirow{3}{*}{\multicolumn{2}{|c|}{$\begin{array}{l}\text { @Participants: } \\
\text { @Age of NAR: } \\
\text { @Sex of NAR: }\end{array}$}} & NAR2 Lindsay Narrator \\
\hline & & 26 \\
\hline & & female \\
\hline @Bg: & ee & \\
\hline *NAR: & & $\begin{array}{l}\text { I myself was quite recently in a pretty dangerous } \\
\text { situation. }\end{array}$ \\
\hline @Eg: & ee & \\
\hline @Bg: & os & \\
\hline *NAR: & & um \# I was here living in Spain. \\
\hline @Eg: & & \\
\hline @Bg: & ocs/da & \\
\hline *NAR: & & and some friends of mine had a yacht. \\
\hline *NAR: & & and one weekend they asked me. \\
\hline *NAR: & & if I wanted to sail with them to Mallorca. \\
\hline @Eg: & $\mathrm{ocs} / \mathrm{da}$ & \\
\hline @Bg: & ee & \\
\hline *NAR: & & $<$ so_> I love sailing $\backslash$. \\
\hline @Eg: & ee & \\
\hline @Bg: & $\mathrm{da}$ & \\
\hline *NAR: & & and $<$ so_> of course I agreed to go/. \\
\hline
\end{tabular}




\section{Montserrat González}

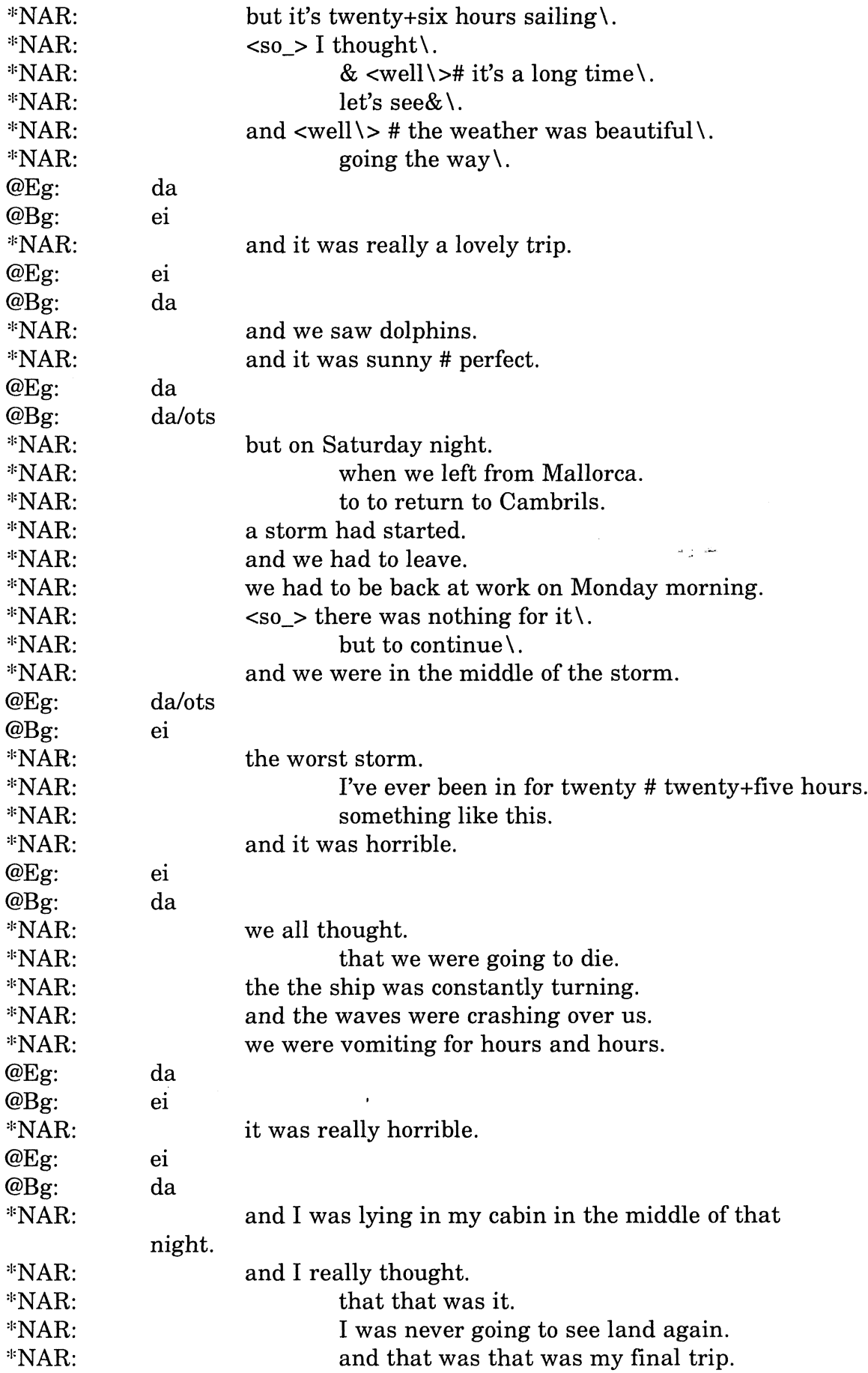




\section{Atención y relato}

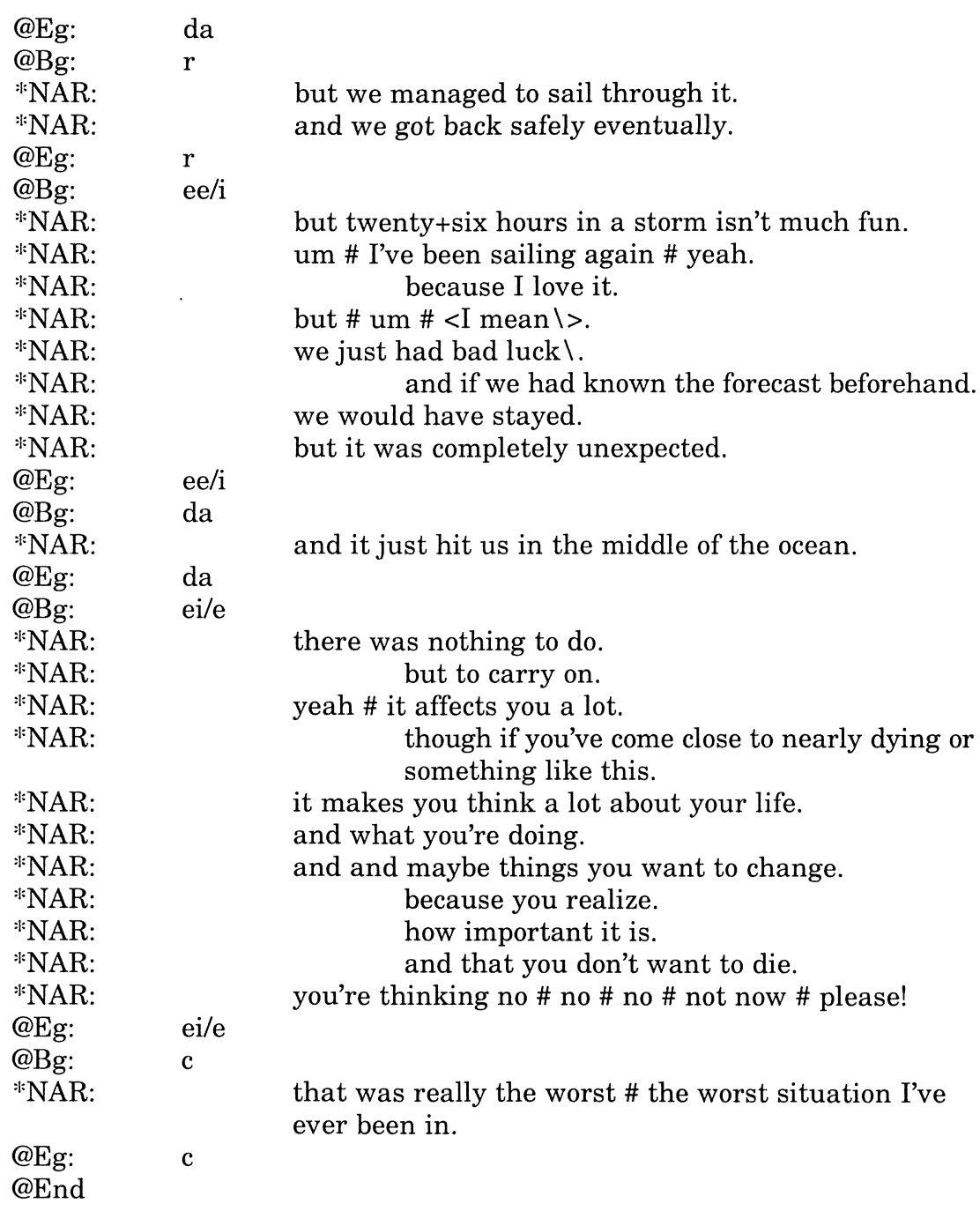

La organización del relato es clausal: cláusulas enlazadas por una conjunción (and) y cláusulas subordinadas, en muchos casos introducidas por una conjunción o forma adverbial. De acuerdo con Labov (1972), las cláusulas propiamente 'narrativas' son solamente aquéllas que incluyen una forma verbal en tiempo pasado y mantienen un estricto orden temporal de los acontecimientos. Veamos la definición que Labov hace de secuencia narrativa ${ }^{7}$, así como del tipo de cláusulas que considera forman su eje central:

"They are independent clauses with verbs in the indicative mood and (in English) one of three tenses: the preterit, the historical present, or the past progressive (...) na- 


\section{Montserrat González}

rrative clauses can be identified by the criterion that they are appropriate answers to the criterial question, 'And then what happened? The sequence of narrative clauses forms the COMPLICATING ACTION." (1982:225)

"A NARRATIVE is then a sequence of two or more narrative clauses, that is, a sequence of clauses separated by one or more temporal junctures.» (1982:226)

En (3) vemos un ejemplo de Labov (1972:361) de una secuencia que contiene tres cláusulas; según criterios labovianos, sólo dos (b y c) se consideran propiamente secuencias narrativas, ya que la primera (a) podría ir después de (b) o (c) sin alterar el orden temporal de los acontecimientos:

a I know a boy named Harry.

b Another boy threw a bottle at him right in the head

$\mathrm{c}$ and he had to get seven stitches.

Labov considera que sólamente las cláusulas principales pueden funcionar como propiamente narrativas, ya que las subordinadas no alteran el orden temporal de los acontecimientos y no llevan, por tanto, el peso de la acción. Llevado al terreno que nos ocupa, lo que se deriva de todo ello es que la información más destacada, la sucesión de los hechos, la presentaría el narrador en las cláusulas principales, mientras que aquélla más secundaria aparecería en las subordinadas. Thompson (1987) y otros autores, Fox (1987), cuestionan la correlación que parece hacer Labov con el concepto anteriormente discutido de foreground, en el primer caso, y de background, en el segundo. De la misma manera, Thompson cuestiona la idea de que las cláusulas principales transmitan la parte más importante del relato, destacando que el criterio de ordenación temporal no tiene por qué igualarse al criterio de 'los hechos más importantes'; ambos deberían funcionar de forma independiente y, en último caso, está en manos del autor el decidir si una cláusula narrativa debe funcionar, o no, como material principal -en un primer plano, el del foreground-o secundario -en un segundo plano, de trasfondo de la acción, el plano del background (1987: 436) ${ }^{8}$. Ciertamente, predicados que aparecen en una secuencia temporal introducidos por una cláusula adverbial (considerada subordinada o dependiente de una principal) pueden llevar a cabo una función 'intensificadora' de la acción. El ejemplo de Thompson (1987: 446), en (4), nos lo clarifica:

(4)

"Only after he stopped smiling and shrieking did he go to Stephanie and hug her.» 


\section{Atención y relato}

Lo que ocurre en la primera cláusula adverbial introduce el primer acontecimiento: parar de sonreír y de temblar. Obsérvese cómo la cláusula principal introduce lo que ocurre a continuación, ordenado temporalmente: ir y abrazarla. Según Thompson, el autor quiere recalcar la intensidad de la reacción de Nim, el personaje que lleva a cabo la acción de sonreír y de temblar, hacia Stephanie y su familia: el abrazo sólo es posible después de que Nim se haya calmado, con lo cual hay una dependencia del segundo hecho sobre el primero, a pesar de existir un orden temporal preestablecido por una marca lingüística adverbial que introduce una cláusula subordinada. La correlación entre forma -gramatical y discursiva- y función presenta, como vemos, indefinición. Desde el punto de vista cognitivo, no parece que las explicaciones puramente estructuralistas ofrezcan respuestas satisfatorias. El argumento cognitivista es que los seres humanos categorizamos los acontecimientos de acuerdo con aquello que nos parece más o menos relevante, y que ciertas construcciones morfosintácticas codifican ciertas categorías de acontecimientos. Hopper y Thompson (1980), por ejemplo, sugieren una explicación funcional a las formas verbales transitivas que aparecen en el relato, al hacer una correlación entre transitividad y partes del relato donde se encuentra la información más destacada (foreground). Según los autores, las cláusulas con verbos transitivos reflejan y ponen en evidencia aquellos hechos que el narrador considera que merecen una atención especial (1980: 54).

El fenómeno discursivo de la referencia anafórica -representado principalmente por el pronombre- ha sido también ampliamente estudiado en relación a cómo los oyentes procesamos el discurso a través de ciertas unidades léxicas que nos lo facilitan. En un estudio de relatos polacos, Flashner (1987) ha demostrado que la referencia gramatical está directamente ligada al contexto discursivo. Así, la utilización de formas nominales o pronominales, o la no aparición de pronombres, tiene una clara relación con la progresión temática: el hablante utiliza determinadas formas de referencia pronominal cuando desea enfatizar un hecho o elemento del relato, su propio punto de vista, o hacer un cambio temático; por el contrario, no utiliza ninguna marca lingüística de referencia cuando hay continuidad temática (de acuerdo con el principio de economía, señala la autora ${ }^{9}$ ). Tomlin (1987) estudia el fenómeno de la referencia en relación a la estructuración segmental del relato, analizando las diversas teorías que existen al respecto: el modelo de cercanía-lejanía del referente, basado en una metodología básicamente cuantitativa, y el modelo que él adopta segmental, de episodios o párrafos. De acuerdo con este último modelo, la alternancia de nombre-pronombre respondería a la capacidad 
limitada de nuestra memoria activada, que se materializa, en el texto, en la forma en que lo estructuramos, en párrafos, segmentos o episodios. Tomlin destaca la conexión directa que hay entre una forma discursiva, esto es, la organización del texto en párrafos, y la capacidad limitada de memoria activada que tenemos los seres humanos. La hipótesis del autor es que los hablantes utilizamos la forma pronominal cuando deseamos mantenernos dentro de un determinado episodio y no deseamos un cambio de atención, y la forma nominal cuando hacemos un cambio, de manera que nuestro foco de atención va estrechamente ligado a la forma en que estructuramos nuestro discurso (1987: 458).

Más adelante reemprenderemos el relato laboviano para ejemplificar la aparición de ciertas unidades léxicas que tienen un papel primordial en cómo procesamos el discurso, los marcadores pragmáticos. La segunda forma de relato oral que ejemplificaremos es una forma ampliamente utilizada en el campo de la psicolingüística y entre los estudiosos de la adquisición de primeras lenguas, en particular. Se trata de la llamada Frog story: Frog, where are you?, creada por Mayer (1969) y explotada en profundidad por Berman y Slobin (1994) para analizar y contrastar la relación forma-función de diversas formas gramaticales y discursivas, mediante estudios comparativos en diversas lenguas. El método, desarrollado por Bamberg (1987), consiste en enseñar a una persona un libro que contiene una serie de 24 dibujos y decirle que la secuencia cuenta una historia. El investigador le pide a la persona en cuestión que le cuente lo que ve, siguiendo el orden de la serie. La historia empieza con una rana que escapa del tarro de cristal en el que está, y un chico y su perro salen a buscarla. En su búsqueda, caen por una ventana, suben y bajan de un árbol, suben a la cima de una roca, y se ven enredados en los cuernos de un ciervo, que arroja al chico al agua de un lago. Al final, cuando el chico sale del agua, descubren a la rana detrás de un tronco. La historia de la rana ha servido a muchos psicolingüistas y estudiosos de la relación entre forma y función del lenguaje (Berman y Slobin 1994, Sebastián y Slobin 1994, Slobin 1996, Fillmore 1996, entre otros) para investigar cómo los hablantes organizamos la información, analizando la progresión temática, la adopción de determinada morfología verbal (en este caso, de verbos de movimiento, en particular), la perspectiva que toma el narrador en el orden en el que ordena las partes de la oración, cómo conecta las oraciones, etc. En definitiva, estudios que implican análisis sintáctico, morfológico, semántico y pragmático.

Slobin (1996), por ejemplo, analiza las escenas donde se produce movimiento, centrándose en la morfología de los verbos utilizados por el narrador de la historia de la rana, en inglés y en español. El autor utiliza 
la clasificación verbal de Talmy (1991: 486) para mostrar que el español, como lengua de enmarcamiento verbal (verb-framed language), utiliza construcciones en las cuales el movimiento y el trayecto forman parte del contenido léxico del verbo, y la manera o no se expresa o se expresa a través de circunstanciales; los verbos son inherentemente direccionales (entrar, bajar, subir, etc.). El inglés, por el contrario, se considera una lengua de enmarcamiento por satélite (satellite-framed language), es decir, utiliza verbos de movimiento que incluyen la manera de la acción que se lleva a término (walk, run, crawl, fly, etc.); las partículas (in, up, to, across, etc.) que acompañan a estos verbos son las que indican el trayecto (Slobin 1996:196) ${ }^{10}$. Veamos en (5) un ejemplo de la historia de la rana (Slobin 1996: 202). Véase la gran cantidad de verbos y satélites utilizados por el narrador.

He [deer] threw him over a cliff into a pond. He [deer] starts running and he tips him off over a cliff into the water.

La utilización de este tipo de verbos hace que los relatos en inglés sean mucho más dinámicos que los relatos en español o cualquier otra lengua románica que utilice verbos de movimiento con contenido léxico. Las unidades que les acompañan, indicando posición y tipo de movimiento, enriquecen la descripción del verbo y elaboran lo que, en términos cognitivistas, se denomina 'trayecto' (Slobin 1996: 198-199)11. Como acertadamente señala Slobin, no es exactamente lo mismo 'Se cayeron al agua' que 'They fell in the water' (p.200). La forma verbal inglesa es mucho más descriptiva, al incluir una preposición que señala direccionalidad (dentro de un 'recipiente'). El estudio de Slobin de actos de movimiento concluye que, en relación a los narradores ingleses, los españoles utilizan muchos menos verbos de movimiento, habiendo por ello de dedicar mucho más tiempo a describir la situación y el trayecto; en inglés, estos elementos pueden, a menudo, inferirse ${ }^{12}$. El ejemplo de (5) muestra, también, cómo la utilización de los verbos acompañados por preposiciones y partículas adverbiales hacen que la atención del oyente se concentre en partes del relato donde hay más acción. Slobin señala que, en español, todo el entorno que proporciona el denominado ground (que incluye acontecimientos, situación y participantes) se ha de explicar, a menudo, mediante cláusulas de relativo («un sendero que conducía al pueblo» - «... walked along a path to the town») (p.206). La utilización de distintas formas lingüísticas tiene una clara repercusión en los estilos retóricos de ambas lenguas: mientras que el narrador del español dedica 
más atención a la descripción del escenario (o ground) y menos a la dinámica de la historia, el narrador inglés proporciona más detalle en cuanto a movimiento y localización de los objectos, enriqueciendo los detalles del trayecto ${ }^{13}$ (1996: 205). Finalmente, el estudio de Slobin muestra que en los relatos españoles hay una utilización más elevada de cláusulas subordinadas adverbiales y de cláusulas de relativo explicativas ${ }^{14}$. Veamos a continuación las marcas lingüísticas 'atencionales' por excelencia, los marcadores pragmáticos. Analistas del discurso y de la comunicación, pragmatistas y estudiosos de los fenómenos lingüísticos que se mueven en el resbaladizo terreno de la pragmasemántica se han volcado, en los últimos años, en su estudio. La gramática tradicional y la lingüística computacional, representadas por dos generaciones de lingüistas, no se han sentido, tampoco, ajenas a su estudio y han investigado, asimismo, su forma y su función en el discurso.

\section{Los marcadores pragmáticos: marcas «atencionales» por excelencia}

Grosz y Sidner (1986) proponen una teoría que plantea que la estructura discursiva está formada por tres componentes, separados pero a la vez interrelacionados: la estructura lingüística formada por la secuencia de oraciones y cláusulas; la estructura intencional, que recoge el propósito del discurso, de los segmentos discursivos y de los participantes en la interacción; y la estructura atencional, que se centra en los elementos, propiedades y relaciones inter e intra segmentales más significativas y relevantes (p. 175-178). De acuerdo con los autores, las marcas lingüísticas que utilizamos los hablantes (modo y aspecto verbal, marcas de referencia y marcadores del discurso, en particular) sirven de puente entre la estructura intencional y la atencional, de forma que, para el interlocutor, resulta evidente el propósito (del texto), la intencionalidad (del hablante) y la delimitación de los distintos segmentos del discurso que interpreta. Según Grosz y Sidner, cuando participamos en una conversación (se podría ampliar a cualquier tipo de intercambio comunicativo verbal), raramente explicitamos, metalingüísticamente, nuestras intenciones y cambios de atención, o 'focus', hacia un determinado elemento o participante. Los marcadores del discurso -o cue phrases ${ }^{15}$ - alertan al oyente que un movimiento o cambio se acerca. En palabras de Grosz y Sidner (1986: 196) estos elementos indican:

1. that a change of attention is imminent;

2. whether the change returns to a previous focus space or creates a new one; 
3. how the intention is related to other intentions;

4. what precedence relationships, if any, are relevant.

Véase el inicio del relato oral catalán de (6). Se trata de un accidente de automóvil que el narrador, Joan, tuvo con una compañera de trabajo. Obsérvese que los tres segmentos -resumen (abstract), orientación (orientation) y evaluación (evaluation) - empiezan con un marcador compuesto.

(6)

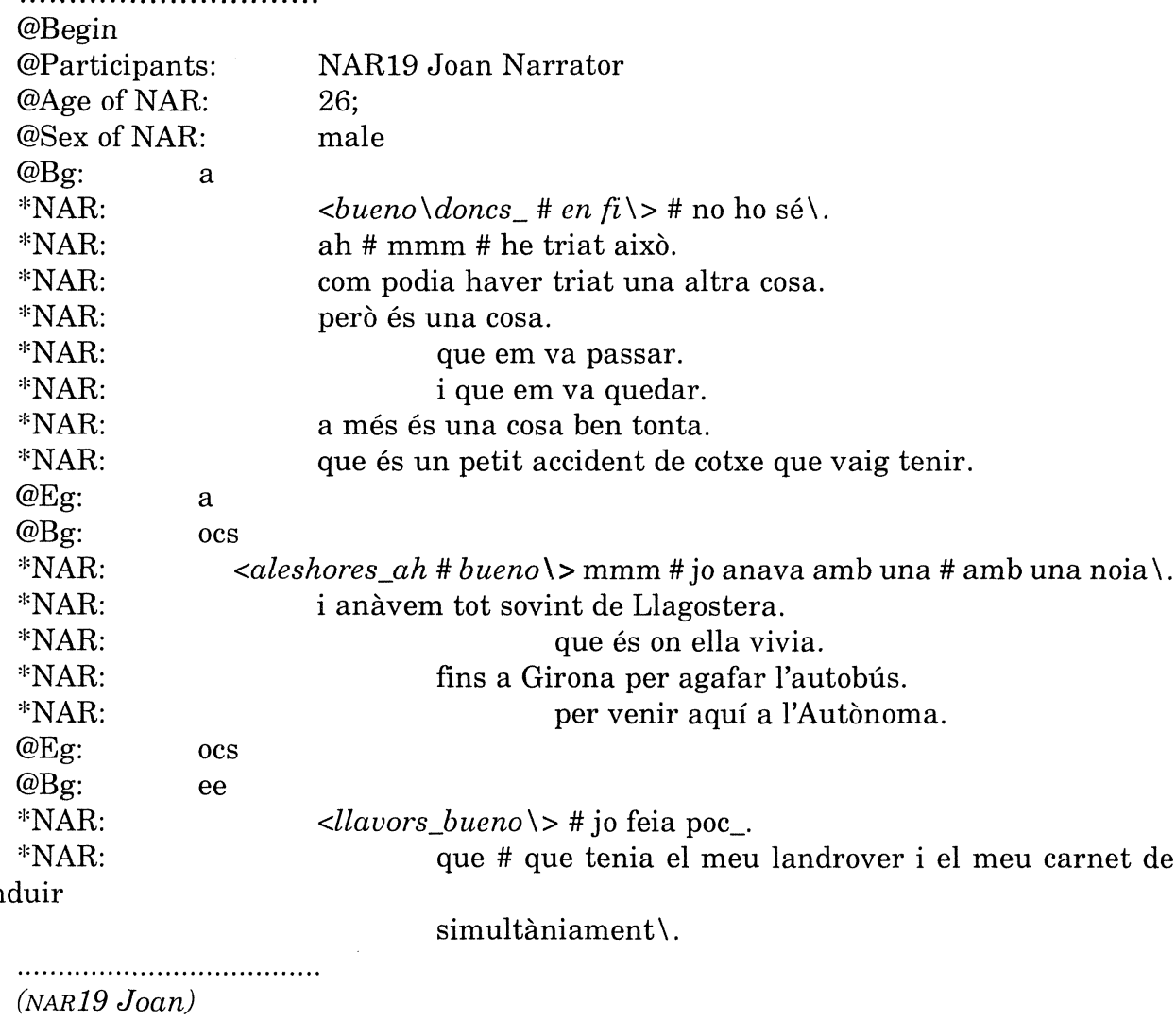

(NAR19 Joan)

La morfología compuesta de los tres marcadores (formados por dos y tres palabras) permite al hablante hacer un cambio atencional importante que implica tres funciones: una de tipo estructural (abriendo los segmentos discursivos), otra de tipo retórico (indicando la intencionalidad del hablante) y otra relacionada con el componente inferencial (situando al interlocutor en el mundo del relato $)^{16}$. Veámoslo con más deta- 
lle. Joan utiliza 'bueno doncs en $f i$ ' (el equivalente léxico sería 'bueno pues en fin'), 'aleshores bueno' ('entonces bueno') y 'llavors bueno' ('entonces bueno') ${ }^{17}$ para abrir o enmarcar los tres primeros segmentos. En el primer y segundo marcador, doncs y ah le sirven también para darle tiempo a pensar lo que va a decir (lo que en inglés llaman 'delaying function'). Finalmente, la tercera unidad del primer marcador ('en $f i$ ') le sirve para introducir una conclusión ('no lo sé'), mientras que la tercera unidad del segundo marcador ('bueno') le sirve para situar al interlocutor en el mundo del relato y orientarlo en términos de personajes involucrados y situación espacial de los acontecimientos. El tercer marcador, que abre una evaluación externa (aportando información que el narrador considera relevante para el interlocutor), sirve para introducir información adicional $^{18}$.

La función de los marcadores pragmáticos como centros deícticos de atención y focalización ha sido también estudiada en relación a las unidades entonativas. Holmqvist y Holsánová (1997) hacen referencia a estudios orales en los cuales el hablante había de narrar (una película, unos hechos, una descripción) y utilizaba estos elementos para saltar de una secuencia a otra, centrando así la atención del interlocutor en un plano distinto (Chafe 1980, Redeker 1990). En estos casos, y en el relato oral en general, el narrador también utiliza los marcadores pragmáticos para recuperar el hilo argumentativo tras una interrupción (del interlocutor, en el caso de relato conversacional), una digresión, o una inclusión de información adicional ${ }^{19}$. Según Chafe (1980), de toda la información que recibimos de nuestro sistema sensorial y nuestra memoria, los hablantes sólo somos capaces de concentrar nuestra atención en determinadas partes, de acuerdo con nuestras necesidades, intereses y objetivos. Nuestro sistema 'atencional' tiene, por lo tanto, una capacidad limitada y selecciona solamente aquella información que posteriormente procesa. Cuando contamos algo de memoria, nuestra atención se mueve de forma secuencial y focalizadora a medida que progresa la historia. En el lenguaje oral, Chafe lo concreta en las unidades entonativas. Los marcadores del discurso, las marcas de referencia y los deícticos, en general, son señales que anuncian un cambio de focus inminente, un movimiento que el hablante difícilmente puede anticipar y predecir (Holmqvist y Holsánová 1997: 224 y 232).

Hemos visto que el significado de las marcas 'atencionales' no es descriptivo o referencial. El ejemplo de Portolés, en (1), lo ponía en evidencia. Su significado es de procesamiento, lo cual significa que su omisión no afecta a las condiciones de verdad del enunciado pero sí a su correcta interpretación. Si hacemos el ejercicio de omitir todos los marcadores 


\section{Atención y relato}

pragmáticos de las secuencias narrativas que hemos ejemplificado, posiblemente entenderemos su contenido, su 'carga proposicional', pero perderemos por el camino toda una serie de elementos pragmáticos que dificultarán el objetivo del narrador, su intencionalidad, adónde pretende llegar, qué desea que inferamos. El discurso será, en definitiva, quizás comprensible pero no 'felicitous' ni 'cooperativo', en términos griceanos.

Las teorías de la Relevancia (Sperber y Wilson 1986), la Gramaticalización y la Subjetivación (Traugott 1995, 1996) no han hecho sino enriquecer el marco conceptual de las marcas lingüísticas 'atencionales'. En términos relevantistas, dichas marcas ponen de manifiesto un entorno cognitivo (Blass 2000), una determinada información, un conocimiento compartido entre los interlocutores. En términos de gramaticalización, el individuo utiliza una serie de elementos que han visto modificado su significado original, que han perdido su carácter referencial total y parcialmente, y han adquirido un significado más pragmático y una categoría distinta, de (más) léxica a (más) funcional, más ligada al discurso, al contexto cognitivo, a la carga expresiva y a la intencionalidad de los participantes en el acto comunicativo. En palabras de Andersen (2000: 34), y citando a Traugott:

"Grammar extends beyond the realms of syntax and semantics and includes the communicative and cognitive facets of a language; it 'encompasses not only phonology, morphosyntax, and truth-functional semantics but also a wide range of inferences that arise out of linguistic form, in other words, linguistic pragmatics, such as focusing, topicalization, and deixis'.» (Andersen 2000: 34)

La subjetivación ha formalizado, lingüísticamente, la presencia del sujeto implicado en el acto comunicativo. Mediante marcas lingüísticas, éste transmite su actitud, su estado y sus intenciones hacia aquello que está contando. Las teorías funcionales, cognitivistas y discursivas han permitido romper barreras entre campos de estudio que, hasta la fecha, han estado estrictamente delimitados. La sintaxis, la semántica, la morfología, la fonología y la pragmática se interrelacionan, conectando forma y función, y se analizan como herramientas que posibilitan lo que, en último término, nos interesa a los participantes de todo acto comunicativo: entendernos.

\section{Notas}

${ }^{1}$ Lo veremos más adelante en la conocida historia de la rana: Frog, where are you? (Mayer 1969), donde los niños tienen que contar qué pasa haciendo un recorrido visual por una serie de viñetas. El estudio exhaustivo de Berman y Slobin (1994) pone de manifiesto diferencias no sólo formales, sino también funcionales y socioculturales. 
2 Segal et al (1991:32) nos aclaran dicha aproximación cognitiva al relato: “A mental model is a mental structure which has properties directly dependent upon the meaning of the text rather than its lexicon or its syntactic form. The deictic shift perspective adds to the concept of the mental model the notion that readers shift their frame of reference for interpreting the sentences being read from a conversational here-and-now situated center to a point or region within the world of the story at or near the events of the story. This region is called the deictic center (Rapaport et al., 1989)."

${ }^{3} \mathrm{Su}$ traducción literal -tema, asunto, tópico- no nos acerca demasiado a su conceptualización cognitiva. La bibliografia anglófona, mayoritaria en este campo de estudio, utiliza ground, foreground y background para referirse a información más o menos 'destacada' relacionada con la situación, los participantes y las circunstancias. Intentaremos parafrasear estos términos, ya que lo importante es que se entienda su significado en relación a la utilización de mecanismos lingüísticos del hablante.

${ }^{4}$ Los autores se refieren a un estudio de textos literarios llevado a cabo por Catherine Chvany (1984).

${ }^{5}$ Los ejemplos de relatos labovianos que muestro pertenecen al estudio llevado a cabo en González (2004). La pregunta inicial de 'sensación de peligro de muerte' fue acortada a 'sensación de peligro', al considerar la autora que, en nuestro entorno sociocultural, mientras que casi todos hemos sentido alguna vez sensación de estar en peligro (un accidente de coche, una excursión, un viaje, haber sufrido un hurto por la calle, etc.), raramente nos hemos sentimos, como en el caso de los adolescentes norteamericanos de Harlem, en peligro de muerte. La entrevista se hizo a veinte adultos (todos licenciados universitarios, entre 25-35 años de edad) nativos de lengua inglesa. La pregunta era: “¿Alguna vez has tenido la sensación de estar en peligro? Cuéntamelo.» En ningún caso hay interrupción por parte del entrevistador, con lo cual, lo que obtenemos es un texto monologado lleno de marcas lingüísticas conversacionales, pero sin los típicos rituales de este género, es decir, sin interrupciones, turnos de habla, cesión de palabra, etc.

${ }^{6}$ Las abreviaturas corresponden a los siguientes segmentos: ee: external evaluation; os: space orientation; ocs: space and characters orientation; ots: space and time orientation; da: developing action; ei: internal evaluation; r: result. Cabe destacar que, en este relato, no encontramos abstract (lo cual resulta muy común en este tipo de relatos).

${ }^{7}$ La traducción literal del término inglés hace siempre referencia al modelo laboviano de narrative.

8 Thompson (1987) ofrece un listado, con ejemplos, de formas gramaticales que pueden considerarse cláusulas subordinadas: un complemento de otro predicado, una cláusula que incluye un verbo no finito, una cláusula introducida por una conjunción adverbial, y una cláusula de relativo (1987: 444)

${ }_{9}$ El principio de economía hace referencia al Principio de Cooperación de Grice (1975). Dicho principio incluye cuatro Máximas Conversacionales (a tener en cuenta por el hablante en todo acto comunicativo): claridad, verdad, relevancia, y cantidad (ésta directamente relacionada con el principio de economía).

${ }^{10}$ Lenguas con verbos-satélite incluyen el holandés, el inglés, el alemán, el polaco, el ruso, el serbocroata, el sueco y el islandés. Con verbos de contenido léxico, el árabe (marroquí), el francés, el hebreo, el italiano, el japonés, el portugués, el español y el turco (Slobin 1996: 205).

11 De acuerdo con Talmy, la escena de un movimiento incluye elementos centrales, tales como la figura, el fondo, el movimiento y el trayecto, y elementos opcionales, tales 


\section{Atención y relato}

como la manera y la causa. Talmy concluye que todas las lenguas formalizan dichos elementos de forma distinta.

12 Partiendo de los componentes de la escena del movimiento de Talmy (1991), Cuenca (2002) ofrece una interesante perspectiva contrastiva de las diferencias gramaticales de varias lenguas, románicas y anglosajonas.

13 Es interesante la observación de Slobin en relación a las consecuencias que dicho fenómeno tiene en la traducción de textos. Al traducir los textos del inglés al español, los traductores a menudo omiten o cambian segmentos del 'trayecto' que enriquecen el relato. Véase el ejemplo que presenta Slobin (1996: 210) de Fowles (1969: 136): "Gradually, he worked his way up to the foot of the bluffs..."; «Poco a poco, fue acercándose hasta el pie de los riscos ..." (que correspondería, literalmente, a: "Gradually, he was approaching the foot of the bluffs...»). Asimismo, Cuenca (2002) aporta datos interesantes en relación a la traducción de textos ingleses al catalán.

14 Sus resultados coinciden con los de González (2004), al hacer el estudio contrastivo de los relatos labovianos en inglés y en catalán.

15 Término ampliamente utilizado por la lingüística computacional, la inteligencia artificial y los estudiosos del procesamiento del discurso. En general, por todos aquéllos que adoptan una perspectiva cognitiva.

16 Diversos modelos de coherencia discursiva cuyo objetivo es dar cuenta de la presencia de los marcadores en el discurso (Schiffrin 1987, Redeker 1991, los más destacados) han puesto en evidencia la polifuncionalidad de estas unidades léxicas. En González (2004) se propone un modelo que abarca los componentes proposicional (o ideacional, en términos de las tres autoras) -dentro del campo más estrictamente semántico- retórico (donde se plasma la intencionalidad del hablante), secuencial (organización en segmentos, párrafos o episodios del discurso) e inferencial (relacionado con todas aquellas marcas lingüísticas que restringen y facilitan las inferencias, el conocimiento compartido, las presuposiciones, etc.) -estos tres últimos dentro del campo más estrictamente pragmático. Dado que nos concentramos en los marcadores relacionados con los componentes pragmáticos, se denominará a estas unidades léxicas marcadores pragmáticos (véase Brinton, 1996, Andersen y Fretheim 2000, Andersen 2000, entre los estudios más destacados de marcadores pragmáticos. En ellos se evidencia su naturaleza cognitiva y su función).

17 La traducción literal léxica de un marcador discursivo no es, en cualquier caso, ni deseable ni recomendable. La traducción al español de los dos adverbios catalanes aleshores y llavors es 'entonces' pero, desde un punto de vista funcional, tal correlación no es exacta. Se ha demostrado que, mientras aleshores es generalmente utilizado por los hablantes para abrir segmentos del discurso y anclar al interlocutor en el mundo del relato (en los componentes secuencial e inferencial, respectivamente, del modelo de coherencia discursiva anteriormente mencionado), llavors se utiliza mayoritariamente para indicar la intencionalidad del hablante y para organizar, temporalmente, los acontecimientos, ideas y situaciones que se dan en el relato. (González 2004, en prensa).

18 Cabe destacar, entre los narradores catalanes, la utilización generalizada de bue no en lugar del equivalente léxico catalán bé, a pesar de no ser una palabra considerada 'normativa' en la gramática y diccionarios catalanes. Se trata de un préstamo lingüístico del español con fines retóricos muy diferenciados del bien o bé catalán (véase estudios al respecto en González 1998 y 2004). Obsérvese cómo, al traducirlo al o desde el inglés, su único equivalente léxico - well-hace que se pierda toda esta valiosa información.

19 En inglés, so es la unidad más utilizada. En español y en catalán, bueno. 


\section{Referencias bibliográficas}

Andersen, G. (2000): Pragmatic Markers and Sociolinguistic Variation. Pragmatics and Beyond New Series 84. Amsterdam/Philadelphia: John Benjamins.

Bamberg, M. y Reilly, J. (1996): «Emotion, Narrative, and Affect: How Children Discover the Relationship Between What to Say and How to Say it.» En Slobin, D.I. et al. (eds.): Social Interaction, Social Context, and Language. Mahwah, NJ: Lawrence Erlbaum, 329-341.

Bamberg, M. y Marchman, V. (1991): «Binding and Unfolding: Towards the Linguistic Construction of Narrative Discourse.» Discourse Processes 14, 277-305.

Berman, R.A. y Slobin, D.E. (eds.) (1994): Relating Events in Narrative. A Crosslinguistic Developmental Study. Hillsdale, NJ: Lawrence Erlbaum.

Blass, R. (2000): «Particles, Propositional Attitude and Mutual Manifestness.» En Andersen, G. y Fretheim, T. (eds.): Pragmatic Markers and Propositional Attitude. Pragmatics and Beyond New Series 79. Amsterdam/Philadelphia: John Benjamins, 39-52.

BRINTON, L.J. (1996): Pragmatic Markers in English. Grammaticalization and Discourse Functions. Berlin/New York: Mouton de Gruyter.

CuencA, M. J. y Hilferty, J.(1999): Introducción a la Lingüística Cognitiva. Barcelona: Ariel.

CuencA, M. J. (2002): "Análisi contrastiva, lingüística aplicada i lingüística teòrica.» En Estudis de llengua i literatura catalanes/XLV. Miscel-lània Joan Veny. Barcelona: Publicacions de l'Abadia de Montserrat, 11-31.

Chafe, W.L. (1980): "The Deployment of Consciousness in the Production of a Narrative." En W.L. Chafe (ed.): The Pear Stories: Cognitive, Cultural, and Linguistic Aspects of Narrative Production. Norwood, NJ: Ablex.

FIllmoRE, C.J. (1996): «The Pragmatics of Constructions.» En Slobin, D.I. et al. (eds.): Social Interaction, Social Context, and Language. Mahwah, NJ: Lawrence Erlbaum, 5369.

FlashneR, V. (1987): “The Grammatical Marking of Theme.» En Tomlin, R.S. (ed.): Coherence and Grounding in Discourse. Amsterdam/Philadelphia: John Benjamins, 131-156.

Fox, B. (1987): “Anaphora in Popular Written English Narratives.» En Tomlin, R.S. (ed.): Coherence and Grounding in Discourse. Amsterdam/Philadelphia: John Benjamins, 157-174.

GonzÁlez, M. (1998): «Be i Bueno. Apunts sobre l'ús dels marcadors discursius». En Lluís Payrató (coord.): oralment. Estudis de Variació Funcional. Barcelona: Publicacions de l'Abadia de Montserrat, 241-257.

GonZÁlez, M. (2004): Pragmatic Markers in Oral Narrative: The Case of English and Catalan. Pragmatics and Beyond New Series 122. Philadelphia/Amsterdam: John Benjamins (en prensa).

GONZÁlEZ, M. (en prensa): “Ús de llavors i aleshores en el 'registre col.loquial». En Actes del 13è Col.loqui Internacional de Llengua i Literatura Catalanes. Barcelona: Publicacions de l'Abadia de Montserrat.

Grosz, B.J. and SidneR, C.L. (1986): "Attention, Intentions, and the Structure of Discourse." En Computational Linguistics 12 (3), 175-204.

HolmqVist, K. y HolsánOVÁ, J. (1997): «Focus Movements and the Internal Images of Spoken Discourse.» En Liebert, W.A., Redeker, G. y WAUGH, L. (eds.): Discourse and Perspective in Cognitive Linguistics. Current Issues in Linguistic Theory 151. Amsterdam/Philadelphia: John Benjamins, 223-246. 


\section{Atención y relato}

Hopper, P. y Thompson, S.A. (1980): «Transitivity in Grammar and Discourse.» Language 56. 251-99.

LABOv, W. y WaletzkY, J. (1967): "Narrative Analysis: Oral Versions of Personal Experience." En HeLm, J. (ed.): Essays on the Verbal and Visual Arts. Seattle: University of Washington Press.

LABOv, W. (1972): "The Transformation of Experience in Narrative Syntax.» En Language in the Inner City. Philadelphia: University of Pennsylvania Press.

LABOV, W. (1982): "Speech Actions and Reactions in Personal Narrative.» En Tannen, D. (ed.): Analyzing Discourse: Text and Talk, Georgetown University Round Table on Languages and Linguistics 1981.Washington, D.C.: Georgetown University Press.

LANGACKER, R. W. (1991): Concept, Image, and Symbol. The Cognitive Basis of Grammar. Cognitive Linguistics Research 1. Berlin/New York: Mouton de Gruyter.

PoRTolés, J. (1998): Marcadores del discurso. Barcelona: Ariel.

REDekeR, G. (1990): «Ideational and Pragmatic Markers of Discourse Structure.» Journal of Pragmatics 14, 367-381.

RedekeR, G. (1991): Linguistic Markers of Discourse Structure: Review of Discourse Markers, by D. Schiffrin. Linguistics 29-6, pp. 1139-1172.

ScheglofF, E.A. (1982): "Discourse as an Interactional Achievement: Some Uses of 'Uh huh' and Other Things that Come Between Sentences.» En Tannen, D. (ed.): Analy. zing Discourse: Text and Talk. Georgetown University Round Table on Languages and Linguistics 1981. Washington, D.C.: Georgetown University Press.

Schiffrin, D. (1987): Discourse Markers. Cambridge University Press.

Sebastian, E. y Slobin, D.I. (1994): «Development of Linguistic Forms: Spanish.» En BeRman, R.A. y Slobin, D.I. (eds.): Relating Events in Narrative. A Crosslinguistic Developmental Study. Hillsdale, NJ: Lawrence Erlbaum.

Segal, E., Duchan, J. y ScotT, P. (1991): "The Role of Interclausal Connectives in Narrative Structuring: Evidence From Adults' Interpretations of Simple Stories." Dis. course Processes 14, 27-54.

SlobIN, D.I. (1996): “Two Ways to Travel: Verbs of Motion in English and Spanish.» En Shibatani, M. y Thompson, S.A. (eds.): Grammatical Constructions. Their Form and Meaning. Oxford: Oxford University Press, 195-219.

Sperber, D. y Wilson, D. (1986): Relevance: Communication and Cognition. Oxford: Basil Blackwell.

Talmy, L. (1991): "Path to Realization: A Typology of Event Conflation.» En Proceedings of the Seventeenth Annual Meeting of the Berkeley Linguistics Society, 480-519.

Thompson, S.A. (1987): “'Subordination' and Narrative Event Structure." En Tomlin, R.S. (ed.): Coherence and Grounding in Discourse. Amsterdam/Philadelphia: John Benjamins, 435-454.

TomLIN, R.S. (1987): “Linguistic Reflections of Cognitive Events.» En Tomlin, R.S. (ed.): Coherence and Grounding in Discourse. Amsterdam/Philadelphia: John Benjamins, 455-479.

Toolan, M. J. (1988): Narrative. A Critical Linguistic Introduction. London/New York: Routledge.

TRAUGOTT, E.C. (1995): Subjectification in Grammaticalization. In Wright, S. and Stein, D. (eds): Subjectivity and Subjectivisation. Cambridge: Cambridge University Press, pp. 31-54.

TraugotT, E.C. (1996): Constructions in Grammaticalization. In Janda, R. and Joseph, B. (eds): A Handbook of Historical Linguistics. Oxford: Basil Blackwell. 